\title{
A THEOREM OF KUMMER'S CONCERNING THE SECOND FACTOR OF THE CLASS NUMBER OF A CYCLOTOMIC FIELD*
}

\author{
BY H. S. VANDIVER
}

Kummer $\dagger$ in a letter to Kronecker dated December 28, 1849 , gave without proof the result that if

$E_{n}=\epsilon^{t} ; t=1+s r^{-2 n}+s^{2} r^{-4 n}+\cdots+s^{(l-3) / 2} r^{-(l-3) n}$,

and

$$
\epsilon=\left(\frac{\left(1-\zeta^{r}\right)\left(1-\zeta^{-r}\right)}{(1-\zeta)\left(1-\zeta^{-1}\right)}\right)^{1 / 2}, \quad \zeta=e^{2 i \pi / l}
$$

and

$$
\prod_{i=1}^{(l-3) / 2} E_{i}^{a i}=\eta^{l}
$$

where $\eta$ is a unit in the cyclotomic field defined by $\zeta$, then each $E_{i}^{a_{i}}$ is the $l$ th power of a unit in the field, the $a$ 's being rational integers. Kummer refers to this result as follows:

"Sehr wichtiger Satz, welcher eine grosse Schwierigkeit hebt."

In the above statement the Kronecker-Hilbert $\ddagger$ symbolic powers are employed; $r$ is a primitive root of the odd prime $l$ and $s$ stands for the substitution $\left(\zeta / \zeta^{r}\right)$. These symbolic powers have the following properties. $\S$ If we denote by $\omega_{k}$ the integer in the field $k(\zeta)$ obtained from the integer $\omega=\omega_{0}$ by means of the substitution $\left(\zeta / \zeta^{r^{k}}\right)$ then we write

* Presented to the Society, New York, March 30, 1929.

† Abhandlungen zur Geschichte der Mathematik, vol. 29 (1910), pp. 84-85.

$\ddagger$ Hilbert, Die Theorie der Algebraischen Zahlkörper, Berichte der Deutschen Mathematiker-Vereinigung, 1894, p. 271.

$\S$ Landau, Vorlesungen über Zahlentheorie, vol. 3, pp. 253-4, proves analogous properties for a field relative to $k(\zeta)$. 


$$
\omega_{0}^{b_{0}} \omega_{1}^{b_{1}} \cdots \omega_{l-2}^{b_{l-2}}=\omega^{F(s)}
$$

the $b$ 's being rational integers and

$$
F(s)=b_{0}+b_{1} s+b_{2} s^{2}+\cdots+b_{l-2} s^{l-2} .
$$

It then follows that

$$
\left(\omega^{F(s)}\right)^{G(s)}=\omega^{F(8) G(s)} .
$$

Employing this relation we shall now prove Kummer's statement. A direct application of it gives

$$
E^{s-r 2 i}=\eta_{i}^{l}
$$

and

$$
E^{s c-r 2 c i}=\eta_{i, c}^{l},
$$

where the $\eta$ 's are units in $k(\zeta)$. Applying the last equation to (1) we may write after employing the substitution $s^{c}$,

$$
\prod_{i=1}^{(l-3) / 2} E_{i} a_{i} r^{2 i c}=\xi^{l},
$$

where $\xi$ is a unit in $k(\zeta)$. We obtain $(l-1) / 2$ equations by taking $c=0,1,2, \cdots,(l-3) / 2$. Raising each equation to the power $r^{-2 c d}$, the $c^{\text {'s }}$ varying as to the particular equation used, we then obtain

$$
E_{d}^{a_{d}} \prod^{a_{i}^{r^{2 c(i-d)}}}=\xi_{c}^{l}
$$

where the product ranges over the values $i=1,2, \cdots$, $(l-3) / 2$ excepting $d$.

Multiplying the $(l-1) / 2$ equations together we obtain

where

$$
E_{d^{a_{d}}}{ }^{(l-1) / 2} \prod E_{j}{ }^{{ }^{k} k}=\xi^{l},
$$

$$
k=\frac{r^{l-1}-1}{r^{2(i-d)}-1} .
$$

The numerator of this fraction is divisible by $l$ and the denominator is prime to $l$ since $(i-d)$ is not divisible by $(l-1)$. Hence we have

$$
E_{d}^{a_{d}}{ }^{(l-1) / 2}=\xi_{d}^{l} .
$$


Raising each side of this equation to the power $l^{\prime}$ where

$$
l^{\prime} \frac{l-1}{2} \equiv 1
$$

we have Kummer's result for $d=1,2, \cdots,(l-3) / 2$.

I think it highly probable that Kummer encountered this question in connection with a problem involving the second factor of the class number of $k(\zeta)$. I shall prove in another article that if the second factor of the class number is divisible by $l$, then (1) holds with not all the $a$ 's divisible by $l$. A somewhat similar result is proved by Hilbert.*

The University of Texas

\section{ON THE RANK EQUATION OF ANY NORMAL DIVISION ALGEBRA†}

BY A. A. ALBERT $\ddagger$

1. Introduction. The different types of normal division algebras which have been discovered up to the present depend upon equations with different groups. It has been thought that, as the rank equation of an algebra is invariant under a change of basal units, the groups of the rank equations of these various types of algebras might serve to show their non-equivalence. This notion is shown to be false here, as the group of the rank equation of any normal division algebra is the symmetric group. In proving this theorem a new theorem in the Hilbert theory of an irreducible polynomial whose coefficients are rational functions, with coefficients in any infinite field $K$, of several parameters is developed.

2. General Theory. We shall first give several presupposed

\footnotetext{
* Loc. cit., pp. 435-437.

$\dagger$ Presented to the Society, December 27, 1928.

$\ddagger$ National Research Fellow.
} 